Discussion Paper No. 10-041

Mutter-Kind-Interaktion im

Säuglingsalter, Familienumgebung und

Entwicklung früher kognitiver und

nicht-kognitiver Fähigkeiten:

Eine prospektive Studie

Dorothea Blomeyer, Manfred Laucht,

Friedhelm Pfeiffer und Karsten Reuß

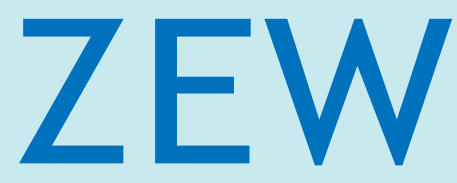

Zentrum für Europäische Wirtschaftsforschung $\mathrm{GmbH}$

Centre for European

Economic Research 
Discussion Paper No. 10-041

\section{Mutter-Kind-Interaktion im Säuglingsalter, Familienumgebung und Entwicklung früher kognitiver und nicht-kognitiver Fähigkeiten: Eine prospektive Studie}

Dorothea Blomeyer, Manfred Laucht, Friedhelm Pfeiffer und Karsten Reuß

Download this ZEW Discussion Paper from our ftp server:

ftp://ftp.zew.de/pub/zew-docs/dp/dp10041.pdf

Die Discussion Papers dienen einer möglichst schnellen Verbreitung von neueren Forschungsarbeiten des ZEW. Die Beiträge liegen in alleiniger Verantwortung der Autoren und stellen nicht notwendigerweise die Meinung des ZEW dar.

Discussion Papers are intended to make results of ZEW research promptly available to other economists in order to encourage discussion and suggestions for revisions. The authors are solely responsible for the contents which do not necessarily represent the opinion of the ZEW. 


\section{Das Wichtigste in Kürze}

In der vorliegenden Studie wird anhand von Daten einer prospektiven Längsschnittstudie (Mannheimer Risikokinderstudie) untersucht, welchen Beitrag die Qualität der Mutter-Kind-Interaktion (MKI) im Säuglingsalter zur Vorhersage kognitiver (Intelligenzquotient, IQ) und nicht-kognitiver Fähigkeiten (Persistenz) bis zum Vorschulalter leistet. Die Mutter-KindDyade wird, dem Ansatz der Interaktionsforscher folgend, sowohl vom Verhalten der Mutter als auch von demjenigen des Kindes und der resultierenden Verhaltensanpassung beeinflusst. Die Mannheimer Risikokinderstudie ist eine prospektive Längsschnittsuntersuchung, die im Jahre 1986 begonnen wurde. Die Mutter-Kind-Interaktion wird mit einer zehnminütigen Videoaufzeichnung einer Pflege- und einer Spielsituation erfasst. Das mütterliche Verhalten wird in acht Dimensionen (Emotion, Zärtlichkeit, Lautäußerungen, verbale Restriktion, Kongruenz / Echtheit, Variabilität, Reaktivität / Sensitivität und Stimulation) beurteilt, das kindliche Verhalten in fünf (Emotion/Mimik, Lautäußerungen, Blickrichtung, Reaktivität, potenzielle Interaktionsbereitschaft).

In der Studie werden ökonometrische Modelle geschätzt, die zusätzlich zu den durch Verhaltensbeobachtung gewonnenen MKI-Maßen die psychosoziale und organische Risikobelastung bei Geburt, die sozio-emotionale Familienumgebung und das Haushaltseinkommen enthalten. Unseren Regressionsanalysen zufolge tragen die beiden betrachteten Interaktionsmerkmale, Feinfühligkeit der Mutter und Reaktivität des Kindes, signifikant zur Prognose des IQ bis zum Vorschulalter bei. Die Feinfühligkeit der Mutter trägt ferner zur Prognose der Persistenz im Vorschulalter bei. Aus methodischer Sicht ergibt sich das wichtigste Resultat unserer Studie, dass die Feinfühligkeit der Mutter in der Dyade, die aus einer zehnminütigen Videoaufzeichnung im Säuglingsalter ermittelt wurde, bereits einen wichtigen Beitrag zur Prognose des IQ zu leisten vermag.

Unsere Ergebnisse verdeutlichen darüber hinaus, dass erhebliche Unterschiede in der Feinfühligkeit der Mütter in der Dyade bestehen. Inhaltlich bedeutet dies, dass die Entwicklung von Fähigkeiten, die in der Dyade ihren Ursprung haben, von Beginn an durch eine hohe Ungleichheit der Entwicklungsbedingungen zwischen den Kindern gekennzeichnet ist. Wichtige mütterliche Verhaltensmuster, die negative Auswirkungen auf die Entwicklung haben, sind Bestrafungen, Einschränkungen und mangelnde Kontingenz im Säugling- und Kleinkindalter. Da sich die Feinfühligkeit von Müttern trainieren lässt, sollten wirksame Hilfen für im frühen Alter benachteiligte Kinder (Kinder, die in der frühen Interaktion wenig Stimulation und Kontingenz erfahren) bereits im Säuglingsalter einsetzen. 


\section{Nontechnical Summary}

This study examines to which extent the quality of mother-child interaction during infancy can predict cognitive (IQ) and noncognitive skills (persistence) until pre-school age using data from the Mannheim Study of Children at Risk. Research suggests that the mother-child dyad is influenced by maternal behaviour, infant behaviour as well as by the resulting motherchild interaction. The Mannheim Study of Children at Risk is a longitudinal study, starting in 1986 when the children are born. The quality of motherchild interaction is captured by a ten minute video of a care and a play situation. Maternal behaviour is broken down into eight dimensions (emotion, tenderness, verbalization, verbal restrictions, congruity/authenticity, variability, reactivity/sensitivity and stimulation), infant behaviour is broken down into five dimensions (emotion/facial expressions, verbalization, viewing direction, reactivity and the potential willingness to interact).

We estimate econometric models that use psychosocial and organic initial risk conditions at birth, socio-emotional family environment and household income as explanatory variables in addition to mother-child interaction. According to our regressions interaction in the dyad, maternal responsiveness and child's reactivity, have a significant impact on child IQ already at pre-school age. Furthermore, maternal responsiveness predicts persistence at pre-school age. From a methodological point of view, our results indicate that maternal responsiveness in the dyad, captured by a 10 -minute video during infancy, already contributes to predicting the IQ later in life.

Moreover, our results demonstrate that maternal responsiveness, an important emotional resource during childhood, varies to a significant degree between families. Thus, children are exposed to mother-child interaction to varying degrees, with inequality-increasing consequences for the formation of cognitive and noncognitive competencies. Important maternal behavioral traits that have a negative impact on competence formation are punishment, restrictions and the lack of contingency, especially during infancy and early childhood. Since maternal responsiveness can be trained, effective policies for disadvantaged children (those children who experience low degrees of contingency and stimulation during infancy and early childhood) should start in infancy. 


\title{
Mutter-Kind-Interaktion im Säuglingsalter, Familienumgebung und Entwicklung früher kognitiver und nicht-kognitiver Fähigkeiten: eine prospektive Studie
}

\author{
Dorothea Blomeyer ${ }^{1}$, Manfred Laucht ${ }^{1}$, Friedhelm Pfeiffer ${ }^{2}$ \\ und Karsten Reuß ${ }^{2}$
}

22. Juni 2010

\section{Zusammenfassung:}

In der vorliegenden Studie wird anhand von Daten einer prospektiven Längsschnittstudie (Mannheimer Risikokinderstudie) untersucht, welchen Beitrag die Qualität der Mutter-Kind-Interaktion (MKI) im Säuglingsalter zur Vorhersage kognitiver (IQ) und nicht-kognitiver Fähigkeiten (Persistenz) bis zum Vorschulalter leistet. Dazu wird ein ökonometrisches Modell verwendet, das zusätzlich zu den durch Verhaltensbeobachtung gewonnenen MKI-Maßen die psychosoziale und organische Risikobelastung bei Geburt, die sozio-emotionale Familienumgebung und das Haushaltseinkommen enthält. Sowohl Merkmale der Mutter (Feinfühligkeit) als auch Merkmale des Kindes (Reaktivität) tragen bedeutsam zur Prognose des IQ und der Persistenz bei. Der Erklärungswert der Interaktionsvariablen wird durch die anderen Einflussfaktoren nur teilweise eingeschränkt.

Stichworte: Frühe Mutter-Kind-Interaktion, mütterliche Feinfühligkeit, Reaktivität des Kindes, Geburtsrisiken, psychosoziale Risiken, Intelligenzentwicklung, Familienumgebung

JEL-Klassifikation: D87, I12, I21, J13

Danksagung: Dieser Beitrag wurde durch die großzügige finanzielle Unterstützung der Leibnizgesellschaft, Bonn des Projektes „Nichtkognitive Fähigkeiten: Erwerb und ökonomische Konsequenzen“ (Pakt für Forschung und Innovation 2008) ermöglicht. Manfred Laucht und Dorothea Blomeyer bedanken sich ferner bei der Deutschen Forschungsgemeinschaft sowie beim Bundesministerium für Bildung und Forschung für die Förderung der Mannheimer Risikokinderstudie. Wir bedanken uns bei Verena Niepel und Pia Pinger für die hilfreichen Kommentare. Noch verbleibende Fehler und Unzulänglichkeiten gehen ausschließlich zu Lasten der Autoren.

Korrespondenz: Friedhelm Pfeiffer, Zentrum für Europäische Wirtschaftsforschung (ZEW), Postfach 1034 43, 68034 Mannheim. Telefon: +49621-1235-150, E-Mail: pfeiffer@zew.de.

${ }^{1}$ Zentralinstitut für Seelische Gesundheit, J 5, D-68159 Mannheim

${ }^{2}$ Zentrum für Europäische Wirtschaftsforschung, L 7,1 D-68161 Mannheim 


\section{Einführung}

Schulerfolg und Persönlichkeitsentwicklung des Kindes werden vielfach von Faktoren bestimmt, die bereits die vorschulische Entwicklung maßgeblich beeinflussen (Cunha und Heckman 2009, Holodynski et al. 2008, Laucht et al. 2000, unter anderen). Eine wichtige Rolle kommt dabei der Qualität der frühen Beziehung zwischen Mutter und Kind zu, die als grundlegend für die Entwicklung von Handlungskompetenzen und seelischer Gesundheit angesehen wird (Field 1981, Greenberg und Crnic 1988, Heckhausen und Heckhausen 2006, unter anderen). Frühe und sehr frühe Erfahrungen in der Dyade und Einflüsse des sozio-emotionalen Umfelds können demnach weit reichende Konsequenzen für die Entwicklung kognitiver und nichtkognitiver Fähigkeiten des Kindes haben.

Die Mutter-Kind-Dyade wird, dem Ansatz der Interaktionsforscher (stellvertretend Ainsworth et al. 1978) folgend, sowohl vom Verhalten der Mutter als auch von demjenigen des Kindes und der resultierenden Verhaltenspassung beeinflusst. Im Rahmen der Mannheimer Risikokinderstudie (Laucht et al. 1997, 2004) wurden Verhaltensbeobachtungen von Müttern mit ihren drei Monate alten Kindern durchgeführt und hinsichtlich verschiedener Interaktionsmerkmale durch trainierte Rater eingeschätzt (Mannheimer Beurteilungsskalen zur Erfassung der Mutter-KindInteraktion im Säuglingsalter [MBS-MKI-S] Esser et al. 1989). Frühere Untersuchungen im Rahmen dieser Studie haben gezeigt, dass die so beurteilte Qualität der Mutter-Kind-Interaktion (MKI) im Säuglingsalter einen wichtigen Beitrag zur Prognose von Verhaltensaufälligkeiten bis ins Schulalter leistet. Unter anderem stellt die Feinfühligkeit einer Mutter in Familien mit hoher psychosozialer Risikobelastung einen Faktor dar, der vor Verhaltensstörungen des Kindes im Schulalter schützt (Laucht et al. 2001, Laucht 2005).

In der vorliegenden Arbeit werden die frühen MKI-Maße gemeinsam mit anderen Frühindikatoren der Mannheimer Risikokinderstudie (Familienumgebung, Haushaltseinkommen) zu einer Analyse der Entwicklung des Intelligenzquotienten (IQ, kognitive Fähigkeit), sowie der Persistenz (P, nicht-kognitive Fähigkeit) verwendet. Dabei beschränkt sich die Arbeit auf die Untersuchung der Entwicklung bis zum Vorschulalter, weil der Intelligenzquotient in diesem Alter bereits eine hohe Stabilität aufweist. So beträgt die Korrelation zwischen dem IQ im Kleinkind- und Vorschulalter in der Mannheimer Risikokinderstudie $\mathrm{r}=0,72$ und zwischen dem Vorschulund dem Schulalter r=0,74 (Blomeyer et al., 2009, S. 641; zur Verstetigung des IQ ab dem Vorschulalter siehe auch Holodynski et al., 2008). 
Die prädiktive Bedeutung der frühen MKI für die Entwicklung der frühen Intelligenz sowie der Persistenz wird hier in einem Modell überprüft, dessen formale Struktur der Technologie des Erwerbs von Fähigkeiten entlehnt ist. Die Kompetenzentwicklung wird als synergetischer und kumulativer Entwicklungsprozess mittels ökonomischer Modelle („Technologie des Erwerbs von Fähigkeiten“) beschrieben und empirisch untersucht (Cunha und Heckman 2009). Cunha et al. (2010) verwenden zur Abschätzung der Höhe der elterlichen Investition in die Fähigkeiten der Kinder eine Stichprobe von 2.209 erstgeboren weißen Kindern von Müttern, die an der National Longitudinal Study of Youth (CNLSY/79) teilgenommen haben. Aufbauend auf ausgewählten Merkmalen der sozio-emotionalen Familienumgebung, darunter die Häufigkeit, mit der die Mutter das Kind lobt, dem Kind vorliest oder Mahlzeiten mit dem Kind einnimmt, wird ein zusammenfassender Indikator der elterlichen Investitionen gebildet. Die verwendeten Merkmale bilden somit Aspekte des mütterlichen Erziehungsverhaltens ab, enthalten aber keine Merkmale des kindlichen Verhaltens in der Interaktion mit der Mutter. Blomeyer et al. (2009) schätzen die Parameter der Technologie des Erwerbs von Fähigkeiten erstmals mit den Daten der Mannheimer Risikokinderstudie vom Säuglings- bis ins frühe Jugendalter. Die elterlichen Investitionen gingen mit einem Maß der sozio-emotionalen Familienumgebung (HOME; Bradley, 1989) ein. Dabei blieb jedoch die Qualität der frühen MKI unberücksichtigt. Ebenso fehlte eine differenzierte Betrachtung von Merkmalen der Familienumgebung.

In unserem Beitrag untersuchen wir erstmals die Zusammenhänge zwischen der Entwicklung in der frühen Kindheit und der Mutter-KindInteraktion im Säuglingsalter unter gleichzeitigem Einbezug spezifischer Merkmale der sozio-emotionalen Familienumgebung und des verfügbaren Haushaltseinkommens. Damit soll eine Forschungslücke in der Literatur zur Technologie des Erwerbs von Fähigkeiten geschlossen werden. Gerade die Säuglingsphase ist durch eine außergewöhnlich hohe Dynamik der Interaktion in der Dyade gekennzeichnet, deren Konsequenzen mangels geeigneter Daten zur Qualität der frühen Interaktion bislang untererforscht sind.

Unser Beitrag ist wie folgt aufgebaut. Im Abschnitt 2 wird die Mannheimer Risikokinderstudie vorgestellt. Abschnitt 3 führt in die psychometrischen Methoden zur Messung der frühen MKI, der Familienumgebung, des IQ und der Persistenz ein. Abschnitt 4 diskutiert die Schätzgleichungen und die Schätzergebnisse. In Abschnitt 5 werden die Schätzergebnisse zusammengefasst und gewürdigt. 


\section{Mannheimer Risikokinderstudie}

Die Mannheimer Risikokinderstudie ist eine prospektive Längsschnittsuntersuchung, die in den Jahren 1986 bis 1988 begonnen wurde. Das Ziel der Studie ist die Analyse der Ursachen und des Verlaufs von Entwicklungsund Verhaltensstörungen. 384 Kinder, die in unterschiedlichem Ausmaß mit psychosozialen und organischen Risiken belastet sind, werden seit der Geburt in ihrer Entwicklung begleitet (Laucht et al. 1997, 2004). Die bisherigen Erhebungen fanden statt, als die Kinder drei Monate, zwei, viereinhalb, acht, elf, fünfzehn und neunzehn Jahre alt waren. Die vorliegende Untersuchung stützt sich auf die ersten drei Erhebungszeitpunkte. Die Stichprobe wurde aus zwei Geburts- und sechs Kinderkliniken der RheinNeckar-Region rekrutiert. Die konsekutiv in die Studie aufgenommenen Kinder mussten die folgenden Einschlusskriterien erfüllen: erstgeborenes Kind, deutschsprachige Eltern, keine Mehrlingsgeburt, keine angeborene schwere Behinderung, Fehlbildung, genetische Abnormalität oder Stoffwechselstörung.

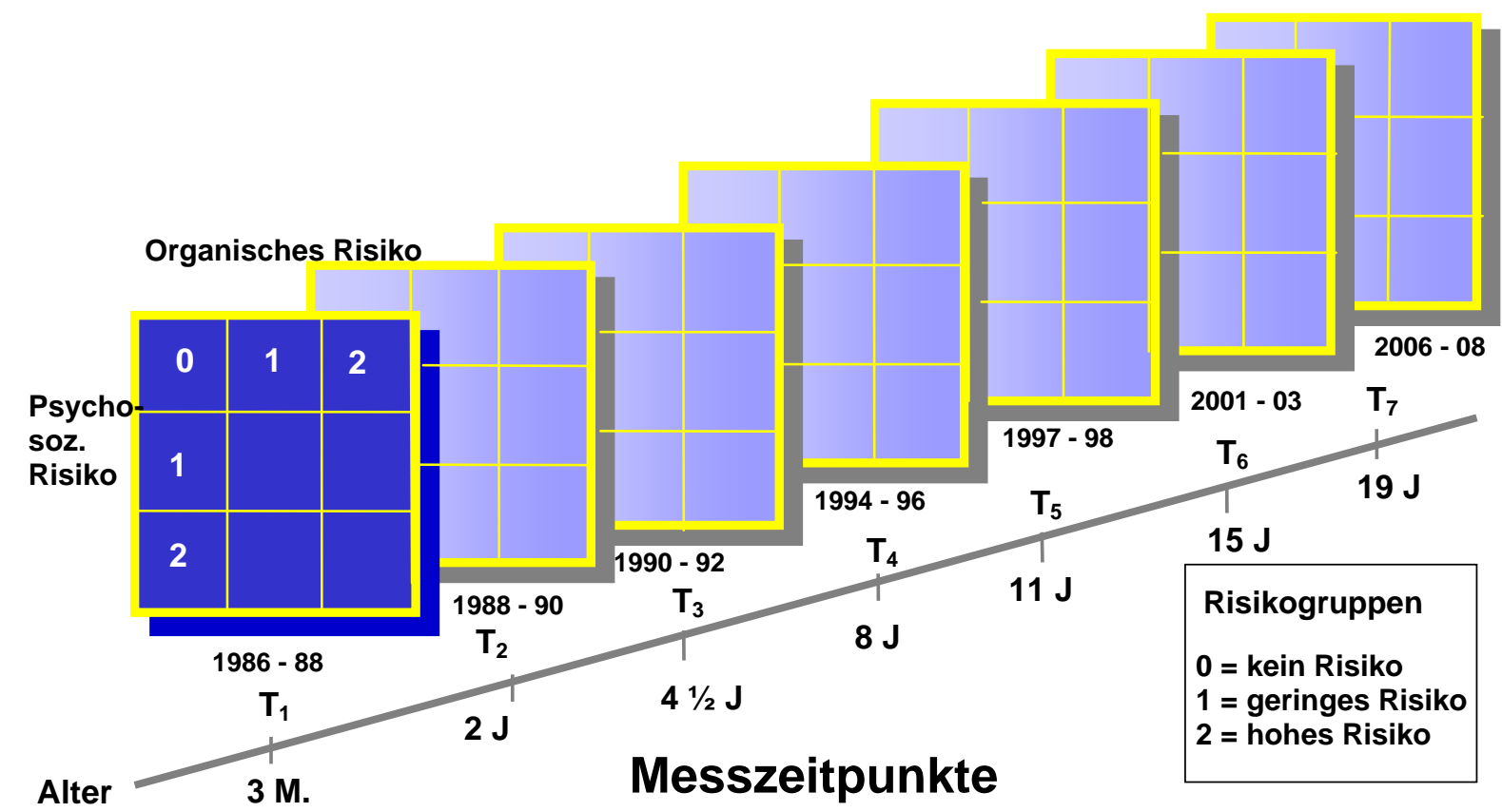

Abbildung 1: Design der Mannheimer Risikokinderstudie (MARS)

Zur Kontrolle bei Geburt bestehender Risiken wurden die Kinder gleichmäßig auf die neun Gruppen eines zwei-faktoriellen Versuchsplans verteilt, in dem das Ausmaß der organischen und psychosozialen Risikobelastung systematisch (in jeweils drei Stufen von keiner bis schwerer Belastung) variiert wurde. Alle Untergruppen dieses Designs sind etwa gleich groß, mit einer leichten Überbesetzung in den Hochrisikogruppen (s. Abbildung 1). 
Aus der Zugehörigkeit zu einer Gruppe der Neunfeldermatrix des organischen und psychosozialen Risikos wird eine, im Folgenden als GEBURTSRISIKO bezeichnete, Maßzahl gebildet, die den höchsten Wert annimmt, wenn kein Risiko vorhanden ist und sich bei einem mittleren Risiko um eine Einheit und bei einem hohen Risiko um zwei Einheiten reduziert. In die vorliegende Auswertung gehen $\mathrm{N}=372$ Kinder ein.

\section{Erfassung der Mutter-Kind-Interaktion (MKI) im Säuglingsalter, der Familienumgebung (HOME) sowie der Intelligenz (IQ) und Persistenz (P) im Entwicklungsverlauf}

\section{Mutter-Kind-Interaktion (MKI-Kind, MKI-Mutter)}

Die Mutter-Kind-Interaktion wird mit einer zehnminütigen Videoaufzeichnung einer Pflege- und einer Spielsituation erfasst. Die Aufnahmen wurden mit zwei Kameras und einer Split-Screen-Technik im Videolabor der Klinik erstellt und mit der Mannheimer Beurteilungsskala zur Erfassung der Mutter-Kind-Interaktion im Säuglingsalter (MBS-MKI-S; Esser et al., 1989) ausgewertet. Diese Methode basiert auf der Einschätzung einminütiger Interaktionssequenzen durch trainierte Rater auf 5-stufigen Skalen: Das mütterliche Verhalten wird in 8 Dimensionen (Emotion, Zärtlichkeit, Lautäußerungen, verbale Restriktion, Kongruenz / Echtheit, Variabilität, Reaktivität / Sensitivität und Stimulation) beurteilt, das kindliche Verhalten in fünf (Emotion/Mimik, Lautäußerungen, Blickrichtung, Reaktivität, potenzielle Interaktionsbereitschaft). Die Interraterreliabilität wurde anhand von 32 Mutter-Kind-Paaren bestimmt und variierte von $r_{s}=.66$ (verbale Restriktion) bis $r_{s}=.97$ (Blickrichtung) mit einer mittleren Übereinstimmung von $\mathrm{r}_{\mathrm{s}}=.88$. Durch Aufsummierung wurde eine Skala „Feinfühligkeit“ gebildet $(\mathrm{Mw}=313,4, \mathrm{SD}=34,8$, Cronbach’s Alpha=.84), die das mütterliche Verhalten beschreibt, und eine Skala „Reaktivität“ (Mw=160,3; SD=27,7, Cronbach’s Alpha=.88) für das kindliche Verhalten (Esser et al., 1989).

\section{Familienumgebung (HOME, $H$ )}

Die Dimensionen der sozio-emotionalen Familienumgebung werden in der Mannheimer Risikokinderstudie mit dem HOME (Home Observation for Measurement of the Environment, Bradley 1989) erfasst. Der HOME besteht aus 45 dichotom kodierten Items, die in sechs Subskalen aufgeteilt sind. Der Gesamt-HOME, H, ist die Summe aller Einzelitems. Im Alter von 3 Monaten wurde eine auf das frühe Säuglingsalter abgestimmte Version 
des „HOME Inventory for Infants and Toddlers“ (Caldwell und Bradley, 1984) eingesetzt. Gegenüber der Originalversion mit 45 Items bestand diese aus 26 Items (Tabelle 1). Im Alter von zwei Jahren wurden 40 Items eingeschätzt.

\section{Intelligenz (IQ), Persistenz (P)}

Im Alter von 3 Monaten und mit 2 Jahren wurde der IQ mit dem Mental Developmental Index (MDI) der Bayley Skalen der kindlichen Entwicklung erfasst (siehe ausführlich Blomeyer et al. 2010). Bei den viereinhalbjährigen Vorschulkindern setzt sich der IQ-Wert als Summenwert aus dem Columbia Mental Maturity Scale (CMMS) und dem Untertest „Sätzeergänzen“ des Psycholinguistischen Entwicklungstests zusammen. Die Persistenz, P, beschreibt die Fähigkeit, eine Aktivität auch angesichts von Ablenkungen und Hindernissen aufrecht zu erhalten, bzw. weiter zu verfolgen. Im Alter von 4,5 Jahren schätzten die Untersucher die Ausprägung dieser Eigenschaft auf einer 5-stufigen Skala ein, die aus dem Temperamentskonzept der New Yorker Längsschnittstudie (NYLS; Thomas \& Chess, 1977) abgeleitet wurde. Die Beurteilung erfolgte auf der Basis eines Interviews mit den Eltern und von Verhaltensbeobachtungen in vier Situationen an zwei verschiedenen Tagen in vertrauter (Hausbesuch, psychologische Untersuchung) und fremder Umgebung (Klinik, neurologische Untersuchung, EEG-Labor). Alle Rater wurden in der Einschätzung spezifisch geschult, die mittlere Interraterreliabilität lag für die Erhebung im Alter von 2 Jahren bei Kappa = 0,82 (Spannweite: 0,52-1,0).

Zur Vergleichbarkeit werden alle Variablen (MKI-Maße, HOME Skalen, IQ, P sowie das verfügbare Nettoeinkommen pro Kopf in der Familie, EINKOMMEN) auf einen Mittelwert von 0 und eine Standardabweichung von 1 normiert. Tabelle 2 enthält die deskriptiven Merkmale der standardisierten Variablen von MKI-Mutter und MKI-Kind, sowie, zur Veranschaulichung, von zwei ausgewählten Subskalen des HOME (Bereitstellung von geeigneten Spielmaterialien sowie Vermeidung von Einschränkungen und Bestrafung). Ein zunehmendes psychosoziales Risiko geht mit deutlich niedrigeren Werten der Responsivität der Mutter und der Reaktivität des Kindes einher (Tabelle 2). Bei zunehmendem psychosozialem Risiko geht beispielsweise in der Gruppe der Kinder mit geringem organischem Risiko der MKI Mutter von 0,47 auf -0,14 zurück (von 329 auf 308 in den Orginalwerten).

In Tabelle 3 sind die Mittelwerte und Standardabweichungen der standardisierten Variablen von IQ, HOME und EINKOMMEN in Abhängigkeit von 
der Zugehörigkeit zu einer Risikogruppe dargestellt. Schon im Alter von drei Monaten ist ein deutlicher Einfluss der Risikobelastung auf den IQ zu verzeichnen, der sich im weiteren Verlauf der Kindheit verfestigt. Kinder ohne Risikobelastung weisen Mittelwerte zwischen 0,3 und 0,4 (104,5 106 IQ-Punkte) auf, während mit beiden Risiken belastete Kinder einen Mittelwert von -0,6 bis -0,5 (91 - 92,5 IQ-Punkte) aufweisen. Die Varianz der IQ-Werte nimmt mit dem Risiko zu. So kann auch bei hohem (niedrigem) Risiko noch ein hoher (niedriger) IQ erreicht werden. Demnach gibt es Kinder, die trotz widriger Lebensumwelt relativ hohe IQ-Werte erreichen. Ebenso finden sich Kinder, die unter den Belastungen stärker leiden, und weit unterdurchschnittliche IQ Werte aufweisen. Sowohl organisches als psychosoziales Risiko üben einen negativen Einfluss auf den IQ aus. Die unterschiedlichen Risikofaktoren wirken kumulativ.

Ein weiteres Ergebnis der Studie ist, dass der HOME wie auch die Subskalen des HOME eine beachtliche Streuung aufweisen (Tabelle 2, Tabelle 3). Während Kinder mit hohem organischem Risiko tendenziell sogar leicht höhere HOME Scores haben als Kinder ohne organisches Risiko, geht ein hohes psychosoziales Risiko zu allen Erhebungszeitpunkten mit deutlich niedrigeren HOME Werten einher (Tabelle 3). Die niedrigsten Werte finden sich in der Gruppe ohne organisches und mit hohem psychosozialem Risiko. Insbesondere fallen die niedrigen Werte bei den Subskalen Vermeidung von Einschränkungen und Bestrafung in den psychosozialen Risikogruppen auf (Tabelle 2). Inhaltlich bedeutet dies, dass die Kinder in diesen Risikogruppen häufiger als andere Kinder bestraft werden oder sonstige, der Entwicklung wenig förderliche, Einschränkungen in ihrem Alltag hinnehmen müssen. Trotz der signifikanten Subskalenunterschiede verbleibt eine erhebliche individuelle Heterogenität in der Entwicklung. Ähnlich dem HOME verringert sich auch das EINKOMMEN mit zunehmender psychosozialer Risikobelastung.

Tabelle 4 enthält die Ergebnisse von einfachen linearen Korrelationen zwischen MKI-Mutter (Feinfühligkeit), MKI-Kind (Reaktivität), HOME, EINKOMMEN (drei Monate), IQ und Persistenz (P) zu den Erhebungszeitpunkten. Die Korrelation zwischen den beiden Interaktionsmaßen in der Dyade beträgt 0,41 . Dies bestätigt den bereits oben erwähnten Zusammenhang, aber auch die Tatsache, dass die beiden Maße eigenständige Aspekte der Interaktion erfassen. MKI-Mutter korreliert mit dem HOME zu allen Erhebungszeitpunkten deutlich enger als MKI-Kind. Die Korrelationen zwischen MKI-Kind und HOME bzw. EINKOMMEN sind beide sehr niedrig und statistisch nicht von Null verschieden. IQ und P weisen im Vorschulalter eine Korrelation von 0,52 auf. Die zeitliche Autokorrelation 
zwischen dem HOME im Säuglings- und Kleinkindalter liegt bei 0,55. Die zeitliche Autokorrelation zwischen EINKOMMEN im Säuglings- und Kleinkindalter (zwischen dem Kleinkind- dem Vorschulalter) und liegt sogar bei 0,83 $(0,71)$ (nicht in Tabelle 4 enthalten). Die hohen Werte von HOME und EINKOMMEN weisen darauf hin, dass die ökonomische und sozio-emotionale Familienumgebung eines Kindes eine relativ hohe Stetigkeit aufweist, im Guten wie im Schlechten.

\section{Mutter-Kind-Interaktion im Säuglingsalter und frühe Intelligenzentwicklung}

Um den Beitrag der frühen MKI zur Erklärung des IQ im Alter von drei Monaten, zwei und viereinhalb Jahren zu überprüfen, werden Regressionsmethoden verwendet. Zunächst wird für jeden der drei Erhebungszeitpunkte ein lineares Regressionsmodell mit den drei Prädiktoren GEBURTSRISIKO, MKI-Kind und MKI-Mutter geschätzt (siehe Gleichung (1)). Alle drei Variablen wurden im Säuglingsalter erfasst. Die Schätzergebnisse für alle drei Erhebungszeitpunkte sind in Tabelle 5 unter (1) zusammengefasst. Eine strukturelle Interpretation der Koeffizienten der Gleichung im Alter von drei Monaten erscheint uns für diesen Erhebungszeitpunkt nicht möglich, da alle Variablen zur gleichen Zeit erfasst wurden. Dennoch werden die Schätzergebnisse zu Dokumentationszwecken hinzugefügt.

$I Q_{t}=\beta_{t}^{1}$ GEBURTSRISIKO $+\beta_{t}^{2}$ MKIKind $+\beta_{t}^{3}$ MKIMutter $+\varepsilon_{t}$

Die Schätzergebnisse für die Erhebungszeitpunkte zwei und viereinhalb Jahre verdeutlichen, dass die Zugehörigkeit zu einer Risikogruppe, GEBURTSRISIKO sowie die MKI-Mutter einen signifikanten Beitrag zur Prognose des IQ leisten. Das korrigierte Bestimmtheitsmaß (korr. $\mathrm{R}^{2}$ in Tabelle 5) liegt knapp über dem Wert von 0,1. Von daher können die Faktoren GEBURTSRISIKO, MKI-Kind und MKI-Mutter von Gleichung (1) zusammen zwar nur einen relativ kleinen Teil der gesamten Variation des IQ erklären. Die einzelnen Faktoren (mit einer einzigen Ausnahme: MKIKind im Alter von zwei Jahren) klären jedoch signifikant unabhängige Varianzanteile auf. Der Schätzkoeffizient für MKI-Mutter zum Erhebungszeitpunkt zwei Jahre beträgt 0,17. Eine Erhöhung der Feinfühligkeit der Mutter, MKI-Mutter, um eine Standardabweichung erhöht den IQ um 0,17 Standardabweichungen (um 2,55 IQ Punkte $=0,17 * 15$ ). Auch die Reaktivität des Kindes, MKI-Kind, leistet in Gleichung (1) einen Erklärungsbeitrag 
zum IQ, der zum Erhebungszeitpunkt viereinhalb Jahre mit 0,12 (1,8 IQ Punkte) signifikant und am höchsten ist.

In der zweiten Spezifikation, Gleichung (2) werden zusätzlich die Variablen HOME und EINKOMMEN, gemessen im Säuglingsalter, aufgenommen. Gleichung (2) wird nicht für den ersten Erhebungszeitpunkt geschätzt. Die Ergebnisse sind in Tabelle 5 unter (2) nach den beiden Erhebungszeitpunkten geordnet zu finden.

$$
I Q_{t}=\ldots .+\beta_{t}^{4} H O M E_{3 \text { Monate }}+\beta_{t}^{5} \text { EINKOMMEN }_{3 \text { Monate }}+\varepsilon_{t}
$$

Das korrigierte Bestimmtheitsmaß beträgt zum Erhebungszeitpunkt zwei Jahre 0,17, zum Erhebungszeitpunkt viereinhalb Jahre 0,15. Beide Prädiktoren tragen für beide Erhebungszeitpunkte signifikant zur Prognose des IQ bei, wobei der Koeffizient von EINKOMMEN etwas höher ausfällt als der Koeffizient von HOME. Zur zweiten Erhebung liegt der Wert des Schätzkoeffizienten von EINKOMMEN bei 0,15. Ist EINKOMMEN eine Standardabweichung höher, steigt der IQ um 0,15 Standardabweichung oder 2,3 Punkte. Falls sich der HOME im Säuglingsalter um eine Standardabweichung erhöht, nimmt der IQ um 0,12 Standardabweichung oder 1,8 Punkte zu. Die Aufnahme von HOME und EINKOMMEN im Säuglingsalter in die Regressionsgleichung (2) führt aufgrund der Interkorrelation der Variablen zu einer erwartungsgemäßen Reduktion der Koeffizienten von MKI-Mutter und GEBURTSRISIKO, insbesondere für den Erhebungszeitpunkt 4,5 Jahre. Der Koeffizient für MKI-Mutter bleibt zwar positiv, wird aber insignifikant. Dies dürfte darauf zurückzuführen sein, dass der gemeinsame Varianzanteil der Variablen MKI-Mutter und HOME im Säuglingsalter (Merkmale der Mutter) mit dem IQ des Kindes assoziiert ist. Werden beide Variablen zusammen in der Gleichung (2) verwendet, sinkt der partielle prognostische Beitrag von MKI-Mutter.

Dagegen bleibt der Koeffizient von MKI-Kind durch die Aufnahme von HOME und EINKOMMEN unverändert. Zum dritten Erhebungszeitpunkt mit viereinhalb Jahren prognostiziert eine Erhöhung von MKI-Kind um eine Standardabweichung einen Anstieg des IQ um 0,12 Standardabweichungen oder 1,8 Punkte. Die Reaktivität des Kindes in der Dyade trägt somit eigenständig zur Prognose des IQ im Vorschulalter bei. Ein Kind, das in der Dyade im Säuglingsalter aufmerksamer reagiert, mehr vokalisiert oder häufiger den Blickkontakt zur Mutter sucht, weist später einen höheren IQ auf. Die Reaktivität des Kindes im Säuglingsalter hat demnach eigenständige prognostische Eigenschaften. 
In der dritten Spezifikation, Gleichung (3) wird zusätzlich die Variable HOME, gemessen im Alter von zwei Jahren, hinzugefügt. Da die Variable EINKOMMEN in hohem Maße über die Zeit korreliert ist (siehe Abschnitt 3 oben), wird diese Größe nur gemessen im Säuglingsalter verwendet. Gleichung (3) wird nur für den letzten Erhebungszeitpunkt geschätzt. Das Ergebnis ist in Tabelle 5 unter (3) dokumentiert.

$I Q_{t}=\ldots .+\beta_{t}^{6} H O M E_{2 J a h r e}+\varepsilon_{t}$

Das korrigierte Bestimmtheitsmaß steigt von 0,15 auf 0,20. Der geschätzte Koeffizient für HOME im Alter von zwei Jahren ist 0,27 und signifikant. Liegt der HOME-Wert im Alter von zwei Jahren um eine Standardabweichung höher, nimmt der IQ im Vorschulalter um 0,27 Standardabweichungen oder 4,1 Punkte zu. Durch das Hinzufügen des HOME mit zwei Jahren weisen HOME bei 3 Monaten sowie MKI-Mutter keine eigenständig erklärenden Varianzanteile mehr auf. Deren Koeffizienten werden insignifikant. Zusätzlich zu diesen beiden Variablen erklärt HOME mit zwei Jahren jedoch noch weitere Varianz des IQ mit 4 1⁄2 Jahren.

Wir schließen daraus, dass der HOME, gemessen im Alter von zwei Jahren eine zusätzliche prognostische Bedeutung für den IQ mit viereinhalb Jahren besitzt und den Erklärungsbeitrag in Termini des korrigierten $\mathrm{R}^{2}$ deutlich erhöhen kann. Die Korrelation zwischen dem HOME im Alter von drei Monaten und zwei Jahren beträgt 0,55 (siehe Tabelle 3). Verbesserungen der sozio-emotionalen Familienumgebung im Alter von zwei Jahren scheinen demnach noch einen bedeutsamen Einfluss auf den IQ im Vorschulalter auszuüben. Mit den Variablen GEBURTSRISIKO, MKI-Mutter, HOME und EINKOMMEN im Säuglingsalter lässt sich jedoch ebenfalls bereits eine gute Prognosegüte erzielen. Der Koeffizient von MKI-Kind bleibt durch die Aufnahme von HOME in Gleichung (3) nahezu unverändert. Er steigt leicht von 0,12 auf 0,14.

In der abschließenden Gleichung wird, ausgehend von Gleichung (3), der Gesamt-HOME, gemessen im Alter von drei Monaten $(t=0,25)$ und zwei Jahren ( $t=2)$ durch die Subskalen des HOME ersetzt. Gleichung (4) wird für die beiden Erhebungszeitpunkte zwei und viereinhalb Jahre geschätzt. Tabelle 5 zeigt die Schätzergebnisse unter (4). Das korrigierte Bestimmtheitsmaß steigt kaum noch an, so dass die Subskalen des HOME den Erklärungswert des Modells nicht erhöhen können. Die geschätzten Koeffizienten sind aufgrund der Interkorrelation der Subskalen bis auf je eine Ausnahme insignifikant. Im Alter von zwei Jahren ergibt sich für die Subskala Vermeidung von Einschränkungen und Bestrafung, gemessen im Säug- 
lingsalter, ein signifikanter Koeffizient von 0,15. Somit scheint diese Subskala vor allem die mütterliche Feinfühligkeit zu messen und ihren positiven Einfluss für den IQ zu bestätigen. Für den IQ im Alter von viereinhalb Jahren ergibt sich für die Subskala Bereitstellung von geeigneten Spielmaterialien, gemessen im Alter von zwei Jahren, ein signifikanter Koeffizient von sogar 0,19. Nimmt der Wert dieser Skala im Alter von zwei Jahren um eine Standardabweichung zu, erhöht sich der IQ im Vorschulalter um 0,19 Standardabweichungen oder 2,9 Punkte. Elterliche Anregung durch vielseitige geeignete Spielmaterialien scheint daher vor allem im Kleinkindalter wichtig für die Entwicklung des IQ zu sein.

Wir schließen aus den Schätzungen der Gleichung (4), dass diese Subskalen des HOME, gemessen im Alter von zwei Jahren, die hier erstmals zur Erklärung des IQ verwendet werden, jeweils einen eigenständigen Beitrag zur Prognose des IQ im Vorschulalter leisten. Zwar ist der Gesamt-HOME als Summe der Subskalen ein geeignetes Instrument zur Vorhersage des IQ. Jedoch scheinen einige der Subskalen einen größeren Erklärungsbeitrag als andere zu haben, der zudem entwicklungsspezifisch ist. Ersetzt man in der Schätzgleichung (3) den HOME durch seine Subskalen (Schätzgleichung (4)) dann sinkt der Koeffizient von MKI-Kind leicht von 0,14 auf 0,12 ab. Ein Teil der Variation des IQ im Vorschulalter, der durch MKI-Kind erklärt wird, scheint mit den Subskalen des HOME korreliert zu sein.

Diesen Abschnitt beschließend, werden die Regressionsergebnisse für die zu erklärende Variable Persistenz, P, im Vorschulalter diskutiert. Tabelle 6 enthält die Schätzergebnisse für die Gleichungen (1) bis (4). Insgesamt wurden fünf Regressionsgleichungen geschätzt, deren Spezifikationen (rechter Hand Variablen) identisch sind mit den Gleichungen für den IQ. Erinnert sei daran, dass $\mathrm{P}$ eine nichtkognitive Fähigkeit misst, die eine relativ hohe kontemporäre Korrelation von 0,52 mit dem IQ aufweist (Tabelle 4). Die Ergebnisse für die Gleichungen (1) bis (3) ähneln den vorherigen Resultaten, mit zwei Unterschieden. Das GEBURTSRISIKO bleibt signifikant, ebenso wie die Feinfühligkeit der Mutter, MKI-Mutter. Das Hinzufügen des HOME führt dazu, dass der Koeffizient von MKI-Mutter kleiner wird. EINKOMMEN ist insignifikant und, interessanterweise MKI-Kind. Die Reaktivität des Kindes im Säuglingsalter trägt somit nicht zur Prognose der Persistenz im Vorschulalter bei (wohl aber zur Prognose des IQ, vergleiche Tabelle 5). Ferner zeigt sich, dass die Subskalen Vermeidung von Einschränkungen und Bestrafung sowie Gelegenheiten für Abwechslung im Alltag signifikante Beiträge leisten. 


\section{Interpretation und Schlussfolgerungen}

Mit ihren Verhaltensbeobachtungsdaten ermöglicht die Mannheimer Risikokinderstudie einen Einblick in die Qualität der frühen Beziehung zwischen Mutter und Kind. Unseren Regressionsanalysen zufolge tragen die beiden betrachteten Interaktionsmerkmale, Feinfühligkeit der Mutter und Reaktivität des Kindes, signifikant zur Prognose des IQ bis zum Vorschulalter bei. Die Feinfühligkeit der Mutter trägt ferner zur Prognose der Persistenz im Vorschulalter bei. Der relative Beitrag beider Merkmale verändert sich über den Zeitraum der Prognose und variiert in Abhängigkeit von der Aufnahme weiterer psychometrischer und ökonomischer Erklärungsgrößen. Werden neben den organischen und psychosozialen Risiken bei Geburt und den Interaktionsskalen (MKI) auch die Familienumgebung (HOME) und das Haushaltseinkommen berücksichtigt, wird der Koeffizient der mütterlichen Feinfühligkeit insignifikant, wobei der gesamte Erklärungsbeitrag des Modells (korr. $\mathrm{R}^{2}$ ) leicht ansteigt. Das ist keineswegs überraschend, da HOME und Feinfühligkeit der Mutter bedeutsame gemeinsame Varianzanteile besitzen. Aus methodischer Sicht ergibt sich das erstaunliche Resultat unserer Studie, dass die Feinfühligkeit der Mutter in der Dyade, die aus einer 10-minütigen Videoaufzeichnung im Säuglingsalter ermittelt wurde, bereits einen wichtigen Beitrag zur Prognose des IQ zu leisten vermag.

Damit stehen unsere Befunde im Einklang mit der Literatur zur Bedeutung der Feinfühligkeit der Mutter in der Interaktion für die Kindesentwicklung (NICHD Early Child Care Research Network 2003, Cunha et al. 2010). Darüber hinaus konnte gezeigt werden, dass die durch Verhaltensbeobachtung gemessene Reaktivität des Säuglings in der Dyade zur Prognose des IQ beiträgt. Der Erklärungsgehalt dieser Variable wird durch andere Größen kaum eingeschränkt. MKI-Kind und HOME konkurrieren nicht, da das Interaktionsmaß vor allem Merkmale des Kindes erfasst, darunter angeborene kognitive Fähigkeiten und das kindliche Temperament. Während diese Fähigkeiten zur Vorhersage des IQ beitragen, stellt die mütterliche Feinfühligkeit in der Dyade einen bedeutsamen Prädiktor der Persistenz dar.

Unsere Ergebnisse verdeutlichen darüber hinaus, dass erhebliche Unterschiede in der Feinfühligkeit der Mutter in der Dyade bestehen. Inhaltlich bedeutet dies, dass die Entwicklung von Fähigkeiten, die in der Dyade ihren Ursprung haben, von Beginn an durch eine hohe Ungleichheit der Entwicklungsbedingungen zwischen den Kindern gekennzeichnet ist. Wichtige mütterliche Verhaltensmuster im Umgang mit Säuglingen und Kleinkindern, die negative Auswirkungen auf die Entwicklung haben, sind Bestrafungen, Einschränkungen und mangelnde Kontingenz. Pfeiffer und Reuß 
(2008) untersuchen die langfristigen Konsequenzen dieser frühen Ungleichheit für den Schul- und Arbeitsmarkterfolg in Deutschland. Demnach ist die Summe der Investitionen in Familie und Schule bis zum Alter von 15 Jahren bei relativ guten SchülerInnen fast siebenmal so hoch wie bei den relativ schlechten (Pfeiffer, 2010). Da Interventionsstudien (Clarke et al. 1979, Riksen-Walraven 1978) nahe legen, dass sich die Feinfühligkeit von Müttern trainieren lässt, sollten wirksame Hilfen für im frühen Alter benachteiligte Kinder (Kinder, die in der frühen Interaktion wenig Stimulation und Kontingenz erfahren) bereits im Säuglingsalter einsetzen.

Einschränkend fügen wir hinzu, dass sich die vorliegende Untersuchung auf eine Stichprobe von nur 372 Beobachtungen der Mannheimer Risikokinderstudie stützt. Damit wird zwar die Datenbasis der 384 Kinder dieser Studie annähernd voll ausgeschöpft. Jedoch sind die Teilnehmer der Mannheimer Risikokinderstudie nicht repräsentativ für die Gesamtbevölkerung, da es sich um eine mit Risikokindern angereicherte Stichprobe handelt, die zudem auf erstgeborene Kinder mit deutschsprachigen Eltern beschränkt ist. Insofern beansprucht die vorliegende Untersuchung keine Repräsentativität für die Interaktionsqualität in der Dyade und der Familienumgebungen, in denen Kinder in Deutschland aufwachsen.

Eine weitere Einschränkung könnte sich aus der Methode zur Erfassung der MKI ergeben. Die Messungen basieren auf einer Verhaltensbeobachtung in einer Laborsituation, die 10 Minuten dauerte. Zwar sind die zentralen Merkmale von Mutter und Kind in face-to-face Interaktionen von einer erheblichen Dynamik gekennzeichnet und basieren vielfach auf unbewussten Handlungen (Papousek und Papousek, 1982). Ob eine Verhaltensprobe in dieser Größenordnung und unter den genannten Kontextbedingungen die Qualität der frühen Mutter-Kind-Interaktion ausreichend valide und repräsentativ abbildet, ist bislang nicht hinreichend geklärt. Immerhin könnten die Mütter in den zehn Minuten versuchen, nur sozial erwünschte Reaktionen zu zeigen.

Insgesamt betrachtet, ermöglicht die vorliegende Untersuchung eine erste Einschätzung der Bedeutung früher Interaktionsmerkmale der Dyade und ihrer Konsequenzen für die Entwicklung kognitiver und nicht-kognitiver Fähigkeiten des Kindes, die in großen, repräsentativ angelegten Erhebungen bislang noch nicht möglich ist. Es bedarf daher weitergehender Forschungen, um die Folgen dieser vielfach unbeobachteten, aber entwicklungsrelevanten Erfahrungen im Säuglingsalter für den Schul- und Arbeitsmarkterfolg besser beurteilen zu können. 


\section{Literaturverzeichnis}

Ainsworth M.D., M.C. Blehar, E. Waters und S. Wall (1978): Patterns of Attachment: a psychological study of the strange situation. Hillsdale, NJ: Erlbaum.

Blomeyer, D., K. Coneus, M. Laucht und F. Pfeiffer (2009): Initial Risk Matrix, Home Resources, Ability Development and Children's Achievement. Journal of the European Economic Association, 7 (2-3), 638-648.

Blomeyer, D., K. Coneus, M. Laucht und F. Pfeiffer (2010): Early Life Adversity, Home Environment and Children's Competence Development, Manuskript Mannheim.

Bradley, R. H. (1989): The Use of the HOME Inventory in Longitudinal Studies of Child Development. In: M. H. Bornstein, und N. A. Krasnegar (Hrsg.), Stability and continuity in mental development: Behavioral and biological perspectives. Hillsdale, New Jersey: Lawrence Erlbaum, 191215.

Caldwell B.M. und R.H. Bradley (1984): Home observation for measurement of the environment. Little Rock, University of Arkansas.

Clarke-Stewart, K. A., L. P. VanderStoep und G. A. Killian (1979): Analysis and Replications of Mother-Child Relations at Two Years of Age. Child Development, 50 (3), 777-793.

Cunha, F. und J. J. Heckman (2009): The Economics and Psychology of Inequality and Human Development. Journal of the European Economic Association, 7 (2-3), 320-364.

Cunha, F., J. J. Heckman und S. Schennach (erscheint demnächst): Estimating the Technology of Cognitive and Noncognitive Skill Formation, Econometrica.

Esser, G., A. Scheven, A. Petrova, M. Laucht und M.H. Schmidt (1989): Mannheimer Beurteilungsskala zur Erfassung der Mutter-KindInteraktion im Säuglingsalter (MBS-MKI-S), Zeitschrift für Kinder- und Jugendpsychiatrie, 17, 185-193.

Field, T. M. (1981): Infant Gaze Aversion and Heart Rate During Face to Face Interactions. Infant Behavior and Development, 4, 307-315.

Greenberg, M. T. und K. A. Crnic (1988): Longitudinal Predictors of Developmental Status and Social Interaction in Premature and Full-Term Infants at Age Two. Child Development, 59, 554-570.

Heckhausen, J. und H. Heckhausen (2006): Motivation und Entwicklung. In: J. Heckhausen und H. Heckhausen (Hrsg.): Motivation und Handeln. Berlin, Springer, Kapitel 15.

Holodynski, M., F. Stallmann und D. Seeger (2008): Entwicklung als soziokultureller Lernprozess: Die Bildungsbedeutung von Bezugspersonen für Kinder. In: T. Apolte und A. Funcke (Hrsg.): Frühkindliche Bildung 
und Betreuung - Reformen aus ökonomischer, pädagogischer und psychologischer Perspektive. Baden-Baden, Nomos, Europäische Schriften zu Staat und Wirtschaft, Band23.

Laucht, M. (2005): Die langfristigen Folgen früher Entwicklungsrisiken: Ergebnisse der Mannheimer Längsschnittstudie zu Risiko- und Schutzfaktoren. In P. Arnoldy und B. Traub (Hrsg.), Sprachentwicklungsstörungen früh erkennen und behandeln, Loeper, Karlsruhe, 169-183.

Laucht, M., G. Esser und M. H. Schmidt (1997): Developmental Outcome of Infants Born with Biological and Psychosocial Risks. Journal of Child Psychology and Psychiatry, 38 (7), 843-854.

Laucht, M., G. Esser und M. H. Schmidt (2000): Längsschnittforschung zur Entwicklungsepidemiologie psychischer Störungen: Zielsetzung, Konzeption und zentrale Ergebnisse der Mannheimer Risikokinderstudie. Zeitschrift für Klinische Psychologie und Psychotherapie, 29 (4), 246-262.

Laucht M, G. Esser und M. H. Schmidt (2001): Differential development of infants at risk for psychopathology: The moderating role of early maternal responsivity. Developmental Medicine and Child Neurology, 43, 292-300

Laucht, M., M. H. Schmidt und G. Esser (2004): The Development of Atrisk Children in Early Life. Educational and Child Psychology, 21 (1), 20-31.

NICHD Early Child Care Research Network (2003): Does Amount of Time Spent in Child Care Predict Socioemotional Adjustment During the Transition to Kindergarten? Child Development, 74, 976-1005.

Papousek, H. und M. Papousek (1982): Die Rolle der sozialen Interaktionen in der psychischen Entwicklung und Pathogenese von Entwicklungsstörungen im Säuglingsalter. In: Nissen, G. (Hrsg.): Psychiatrie des Säuglings- und frühen Kindesalter. Wien, Huber.

Pfeiffer, F. (2010): Entwicklung und Ungleichheit von Fähigkeiten: Anmerkungen aus ökonomischer Sicht. In: H.-H. Krüger, U. Rabe-Kleberg, R.-T. Kramer und J. Bude (Hrsg.): Bildungsungleichheit revisited. Bildung und soziale Ungleichheit vom Kindergarten bis zur Hochschule. Wiesbaden, Verlag für Sozialwissenschaften, Studien zur Schul- und Bildungsforschung, 30, 25-44.

Pfeiffer, F. und K. Reuß (2008): Age-dependent Skill Formation and Returns to Education. Labour Economics, 15 (4), 631-46.

Riksen-Walraven, J.M. (1978): Effects of caregiver behavior on habituation rate and self-efficacy in infants. International Journal of Behavioral Development, 1, 105-130.

Thomas, Alexander, and Stella Chess (1977): Temperament and Development, Brunner/Mazel, New York. 
Tabelle 1: Subskalen und Itemaufteilung des HOME Inventory for Infants and Toddlers in der Original- und der MARS-Version mit 3 Monaten und 2 Jahren

\begin{tabular}{lccc}
\hline \hline & $\begin{array}{c}\text { Ori- } \\
\text { ginal }\end{array}$ & MARS & MARS \\
& 0-3J. & 3 Monate & 2 Jahre \\
\hline Subskalen & & & \\
I Emotionale und verbale Responsivität der Mutter & 11 & 10 & 11 \\
II Vermeidung von Einschränkung und Bestrafung & 8 & 5 & 8 \\
III Stabilität und Gestaltung der Umgebung & 6 & 4 & 6 \\
IV Bereitstellung geeigneten Spielmaterials & 9 & 1 & 8 \\
V Mütterliches Interesse am Kind & 6 & 2 & 2 \\
VI Gelegenheit für Abwechslung im Alltag & 5 & 4 & 5 \\
\hline Gesamt & 45 & 26 & 40 \\
\hline \hline
\end{tabular}


Tabelle 2: Mittelwerte (SD in Klammern) der MKI-Skalen, der HOME Subskalen Spielmaterialen und Vermeidung von Einschränkungen in den Zellen der Risikomatrix mit drei Monaten $(0,25)$ und zwei Jahren (2)

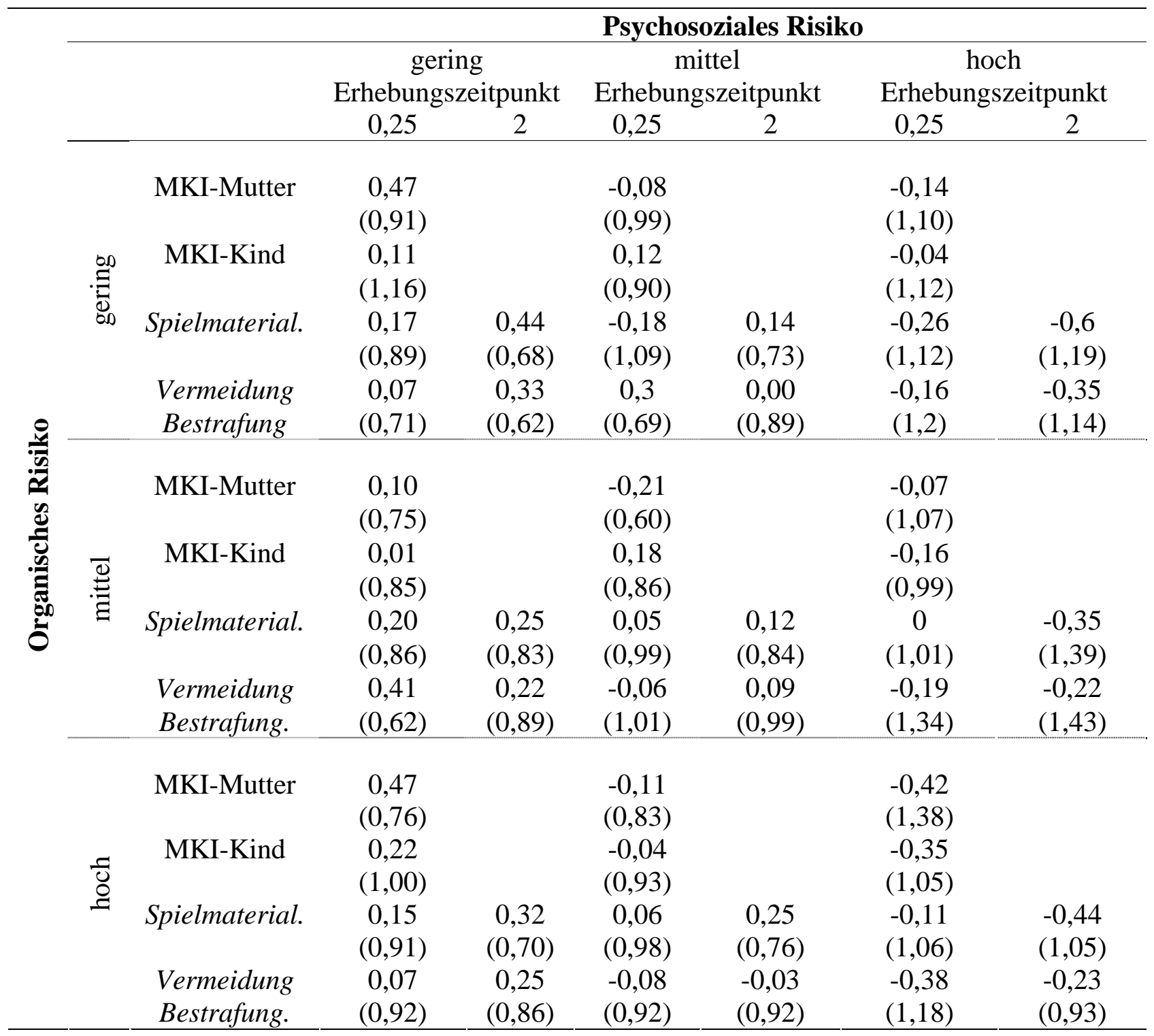

Mannheimer Risikokinderstudie, Stichprobe von 372 Beobachtungen; alle Werte wurden für jeden Erhebungszeitpunkt auf Mittelwert null und Standardabweichung 1 normiert, SD: Standardabweichung. 
Tabelle 3: Mittelwerte (SD in Klammern) von IQ, HOME, Pro-KopfEinkommen im Haushalt mit drei Monaten $(0,25)$, zwei Jahren $(2,00)$ und viereinhalb Jahren $(4,50)$

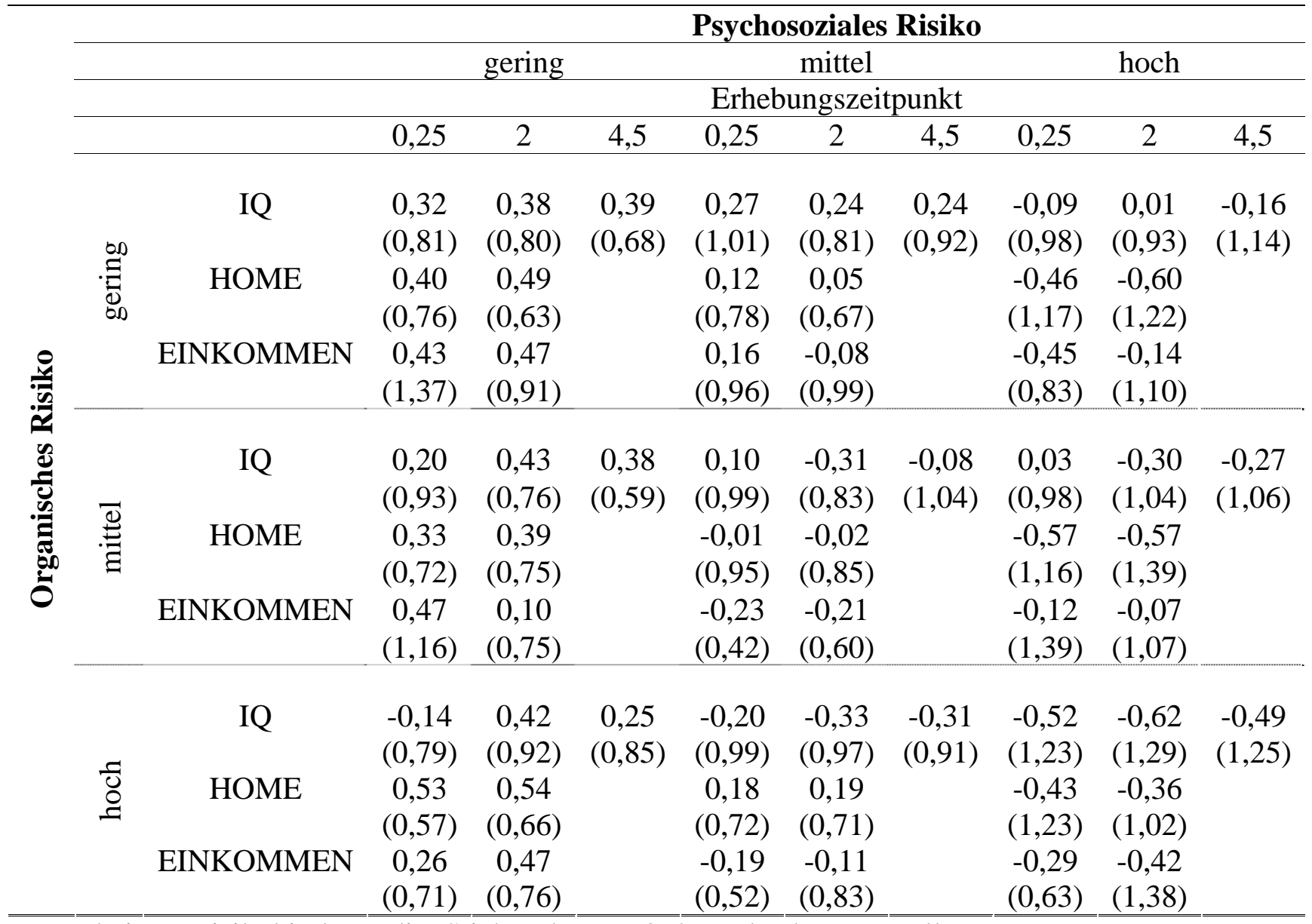

Mannheimer Risikokinderstudie, Stichprobe von 372 Beobachtungen; alle Werte wurden für jeden Erhebungszeitpunkt auf Mittelwert null und Standardabweichung 1 normiert, SD: Standardabweichung, 
Tabelle 4: Korrelationsanalyse MKI-Mutter, MKI-Kind, HOME (H), IQ, Persistenz (P)

\begin{tabular}{|c|c|c|c|c|c|c|c|c|c|}
\hline \multicolumn{2}{|c|}{ Erhebungszeitpunkt } & \multicolumn{4}{|c|}{ 0,25 Jahre } & \multicolumn{2}{|c|}{2 Jahre } & \multicolumn{2}{|c|}{ 4,5 Jahre } \\
\hline & & $\begin{array}{l}\text { MKI- } \\
\text { Mutter }\end{array}$ & $\begin{array}{l}\text { MKI- } \\
\text { Kind }\end{array}$ & IQ & $\mathrm{H}$ & IQ & $\mathrm{H}$ & IQ & $\mathrm{P}$ \\
\hline \multirow{5}{*}{$\begin{array}{c}0,25 \\
\text { Jahre }\end{array}$} & IQ & & & & 0,25 & 0,35 & 0,21 & 0,29 & 0,24 \\
\hline & $\mathrm{H}$ & & & & & 0,25 & 0,55 & 0,24 & 0,26 \\
\hline & MKI-Mutter & & 0,41 & 0,19 & 0,26 & 0,25 & 0,26 & 0,21 & 0,20 \\
\hline & MKI-Kind & & & 0,17 & 0,12 & 0,17 & 0,07 & 0,20 & 0,11 \\
\hline & EINKOMMEN & 0,10 & 0,08 & 0,17 & 0,24 & 0,25 & 0,36 & 0,23 & 0,18 \\
\hline \multirow{2}{*}{$\begin{array}{c}2 \\
\text { Jahre }\end{array}$} & IQ & & & & & & 0,36 & 0,73 & 0,58 \\
\hline & $\mathrm{H}$ & & & & & & & 0,32 & 0,30 \\
\hline $\begin{array}{c}4,5 \\
\text { Jahre }\end{array}$ & IQ & & & & & & & & 0,52 \\
\hline
\end{tabular}

Mannheimer Risikokinderstudie, Stichprobe von 372 Beobachtungen. 
Tabelle 5: Regressionsergebnisse für den IQ zu unterschiedlichen Erhebungszeitpunkten

\begin{tabular}{|c|c|c|c|c|c|}
\hline & \multicolumn{3}{|c|}{$\overline{(1)}$} & \multicolumn{2}{|c|}{$(2)$} \\
\hline & IQ $_{0,25}$ & $\mathrm{IQ}_{2}$ & $\mathrm{IQ}_{4,5}$ & $\mathrm{IQ}_{2}$ & $\mathrm{IQ}_{4,5}$ \\
\hline \multirow[t]{2}{*}{ Geburtsrisiko } & $0,20 *$ & $0,28 *$ & $0,25 *$ & $0,22 *$ & $0,20 *$ \\
\hline & $(, 052)$ & $(, 052)$ & $(, 048)$ & $(, 054)$ & $(, 05)$ \\
\hline \multirow[t]{2}{*}{ MKI-Mutter } & $0,11 *$ & $0,17^{*}$ & $0,10 *$ & $0,13^{*}$ & 0,07 \\
\hline & $(, 059)$ & $(, 057)$ & $(, 061)$ & $(, 058)$ & $(, 062)$ \\
\hline \multirow[t]{2}{*}{ MKI-Kind } & $\mathbf{0 , 1 0 *}$ & 0,07 & $0,12 *$ & 0,06 & $0,12 *$ \\
\hline & $(, 054)$ & $(, 051)$ & $(, 053)$ & $(, 049)$ & $(, 053)$ \\
\hline \multirow[t]{2}{*}{ Home $_{0,25}$} & & & & $0,12 *$ & $0,12^{*}$ \\
\hline & & & & $(, 05)$ & $(, 056)$ \\
\hline \multirow[t]{2}{*}{ Einkommen $_{0,25}$} & & & & $0,15^{*}$ & $0,14 *$ \\
\hline & & & & $(, 038)$ & $(, 037)$ \\
\hline \multirow[t]{3}{*}{ Korrigiertes $\mathrm{R}^{2}$} & 0,08 & 0,14 & 0,11 & 0,17 & 0,15 \\
\hline & (3) & & $(4)(t=0,25)$ & & $=2)$ \\
\hline & $\mathrm{IQ}_{4,5}$ & & $\mathrm{IQ}_{2}$ & & \\
\hline \multirow[t]{2}{*}{ GEBURTSRISIKO } & $0,17 *$ & & $0,21 *$ & & \\
\hline & $(, 05)$ & & $(, 054)$ & & \\
\hline \multirow[t]{2}{*}{ MKI-Mutter } & 0,04 & & $0,13 *$ & & \\
\hline & $(, 061)$ & & $(, 059)$ & & \\
\hline \multirow[t]{2}{*}{ MKI-Kind } & $0,14^{*}$ & & 0,07 & & \\
\hline & $(, 052)$ & & $(, 049)$ & & \\
\hline \multirow[t]{2}{*}{$\mathrm{HOME}_{0,25}$} & $-0,01$ & & & & \\
\hline & $(, 058)$ & & & & \\
\hline \multirow[t]{2}{*}{ EINKOMMEN $_{0,25}$} & $0,11 *$ & & $0,15 *$ & & \\
\hline & $(, 034)$ & & $(, 038)$ & & \\
\hline \multirow[t]{2}{*}{$\mathrm{HOME}_{2}$} & $0,27^{*}$ & & & & \\
\hline & $(, 057)$ & & & & \\
\hline \multirow{3}{*}{$\begin{array}{c}\text { Emotionale und verbale } \\
\text { Responsivität der Mut- }_{\text {ter }_{t}}\end{array}$} & & & $-0,04$ & \multirow{3}{*}{\multicolumn{2}{|c|}{$\begin{array}{c}0,05 \\
(, 063)\end{array}$}} \\
\hline & & & $(, 058)$ & & \\
\hline & & & & & \\
\hline \multicolumn{3}{|l|}{ Vermeidung von } & $0,15^{*}$ & \multicolumn{2}{|c|}{0,03} \\
\hline \multicolumn{3}{|l|}{ Einschränkungen $_{t}$} & $(, 045)$ & \multicolumn{2}{|c|}{$(, 061)$} \\
\hline \multirow{2}{*}{\multicolumn{3}{|c|}{$\begin{array}{l}\text { Stabilität und Gestal- } \\
\text { tung der Umgebung }\end{array}$}} & 0,01 & \multicolumn{2}{|c|}{0,04} \\
\hline & & & $(, 052)$ & \multicolumn{2}{|c|}{$(, 052)$} \\
\hline \multirow{2}{*}{ Spielmaterialien $_{t}$} & & & 0,05 & \multirow{2}{*}{\multicolumn{2}{|c|}{$\begin{array}{l}\mathbf{0 , 1 9 *} \\
(, 004)\end{array}$}} \\
\hline & & & $(, 052)$ & & \\
\hline \multirow{2}{*}{$\begin{array}{c}\text { Mütterliches Interesse } \\
\text { am Kind }\end{array}$} & & & $-0,03$ & & \\
\hline & & & $(, 045)$ & & \\
\hline Abwechslung im Alltag. & & & 0,09 & & \\
\hline Abwechsiung im Alitag & & & $(, 060)$ & & \\
\hline Korrigiertes $\mathrm{R}^{2}$ & 0,20 & & 0,19 & & \\
\hline
\end{tabular}

Mannheimer Risikokinderstudie, Stichprobe von 372 Beobachtungen; KQ Schätzungen mit heteroskedastiekonsistenten Standardfehlern; alle Variablen wurden auf Mittelwert 0, Standardabweichung 1 normiert; * signifikant zum 90 Prozent Niveau. 
Tabelle 6: Regressionsergebnisse für die Persistenz (P) im Alter von 4,5 Jahren

\begin{tabular}{|c|c|c|c|c|c|}
\hline Schätzgleichung & (1) & (2) & (3) & $\begin{array}{c}(4) \\
t=0,25\end{array}$ & $\begin{array}{l}(4) \\
t=2\end{array}$ \\
\hline GEBURTSRISIKO & $\begin{array}{c}\mathbf{0 , 2 5 *} \\
(0,048)\end{array}$ & $\begin{array}{c}\mathbf{0 , 2 0 *} \\
(0,049)\end{array}$ & $\begin{array}{c}\mathbf{0 , 1 9 *} \\
(0,049)\end{array}$ & $\begin{array}{c}\mathbf{0 , 1 8 *} \\
(0,050)\end{array}$ & $\begin{array}{c}\mathbf{0 , 1 8 *} \\
(0,050)\end{array}$ \\
\hline MKI-Mutter & $\begin{array}{c}\mathbf{0 , 1 4} * \\
(0,053)\end{array}$ & $\begin{array}{c}\mathbf{0 , 1 0 *} \\
(0,054)\end{array}$ & $\begin{array}{c}0,08 \\
(0,055)\end{array}$ & $\begin{array}{c}\mathbf{0 , 1 1} * \\
(0,051)\end{array}$ & $\begin{array}{c}\mathbf{0 , 0 9 *} \\
(0,057)\end{array}$ \\
\hline MKI-Kind & $\begin{array}{c}0,02 \\
(0,052)\end{array}$ & $\begin{array}{c}0,02 \\
(0,051)\end{array}$ & $\begin{array}{c}0,03 \\
(0,051)\end{array}$ & $\begin{array}{c}0,02 \\
(0,051)\end{array}$ & $\begin{array}{c}0,02 \\
(0,051)\end{array}$ \\
\hline $\begin{array}{c}\text { HOME, } \\
\text { drei Monate }\end{array}$ & & $\begin{array}{c}\mathbf{0 , 1 6 *} \\
(0,053)\end{array}$ & $\begin{array}{c}0,08 \\
(0,058)\end{array}$ & & $\begin{array}{c}0,09 \\
(0,059)\end{array}$ \\
\hline $\begin{array}{c}\text { EINKOMMEN, } \\
\text { drei Monate } \\
\text { HOME, } \\
\text { zwei Jahre }\end{array}$ & & $\begin{array}{c}0,08 \\
(0,053)\end{array}$ & $\begin{array}{c}0,07 \\
(0,052) \\
\mathbf{0 , 1 7} * \\
(0,06)\end{array}$ & $\begin{array}{c}\mathbf{0 , 0 9 *} \\
(0,053)\end{array}$ & $\begin{array}{c}0,05 \\
(0,052)\end{array}$ \\
\hline Emotionale und verbale & & & & $-0,04$ & $-0,02$ \\
\hline Responsivität der Mutter ${ }_{t}$ & & & & $(0,061)$ & $(0,062)$ \\
\hline Vermeidung von & & & & $0,09 *$ & $\mathbf{0 , 1 1} *$ \\
\hline Einschränkungen $u$. Bestrafung ${ }_{t}$ & & & & $(0,053)$ & $(0,059)$ \\
\hline Stabilität und Gestaltung der & & & & 0,02 & 0,00 \\
\hline Umgebung & & & & $(0,052)$ & $(0,052)$ \\
\hline Spielmaterialien $_{t}$ & & & & $\begin{array}{c}0,00 \\
(0,061)\end{array}$ & $\begin{array}{c}0,08 \\
(0,058)\end{array}$ \\
\hline Mütterliches Interesse am Kind & & & & $\begin{array}{c}0,06 \\
(0,052)\end{array}$ & $\begin{array}{c}0,02 \\
(0,722)\end{array}$ \\
\hline Abwechslung im Alltag & & & & $\begin{array}{c}\mathbf{0 , 1 7 *} \\
(0,066) \\
\end{array}$ & $\begin{array}{c}0,06 \\
(0,057)\end{array}$ \\
\hline Korrigiertes $\mathrm{R}^{2}$ & 0,10 & 0,13 & 0,15 & 0,14 & 0,14 \\
\hline
\end{tabular}

Mannheimer Risikokinderstudie, Stichprobe von 372 Beobachtungen; KQ Schätzungen mit heteroskedastiekonsistenten Standardfehlern; alle Variablen wurden auf Mittelwert 0, Standardabweichung 1 normiert; * signifikant zum 90 Prozent Niveau. 